\title{
A Case of Multisystemic Langerhans Cell Histiocytosis in an Adult
}

\author{
Chng Chiaw Ling \\ Singapore General Hospital
}

\begin{abstract}
A 23-year-old female smoker presented in late 2008 with a large left pneumothorax. She had multiple spontaneous pneumothoraces in 2007 which required pleurectomy. A presumptive diagnosis of lymphangioleiomyomatosis (LAM) was made in 2007 after the high-resolution computed tomography (HRCT) of the thorax revealed bilateral interlobular septal thickening and multiple thin walled cysti: air spaces without lobar predilection, and with preservation of lung volumes.
\end{abstract}

She had new and worsening symptoms of persistent thirst, polydipsia, polyuria, generalized brown papular skin rash and recurrent vulvar ulcerations for one year when she presented in 2008. Endocrinology evaluation revealed central diabetes insipidus (DI) with a normal anterior pituitary axis. MRI of the pituitary gland (Fig. 1) demonstrated classical signs of central DI. A repeat HRCT of the thorax (Fig. 2) revealed extensive cystic lesions of the lungs. The abdominal CT was normal except for multiple focal bony defects at T10 vertebra, both ilia and left trochanter suggesting bone involvement. The diagnosis of Langerhans cell histiocytosis was eventually confirmed on skin and vulvar biopsies demonstrating heavy infiltrates of histiocytes containing abundant foamy to eosinophilic cytoplasm with strong cytoplasmic reaction to CD1a and S100 on immunochemistry staining (Fig.3).

The term Langerhans cell histiocytosis (LCH) encompasses a spectrum of diseases characterized by the proliferation and infiltration of organs by pathological Langerhans $\mathrm{C}:=1 \mathrm{~s}^{1}$. Pulmonary LAM, on the other hand, is a rare disease that almost exclusively affects women of childbearing age. It is characterized by proliferation of atypical smooth muscle-like cells with associated cystic changes in the lungs ${ }^{2}$, and is sometimes associated with tuberous sclerosis complex ${ }^{3,4}$.

In this patient, the initial presentation of recurrent pneumothorax without systemic manifestations of $\mathrm{LCH}$ has led to the initial diagnosis of pulmonary LAM. The multiple thin walled cysts on HRCT of the thorax could suggest possible LAM versus late pulmonary $\mathrm{LCH}$, which is unusual for this patient, considering her young age. However, the appearance of additional systemic clinical features associated with $\mathrm{LCH}$ eventually prompted reexamination of the initial diagnosis. The involvement of
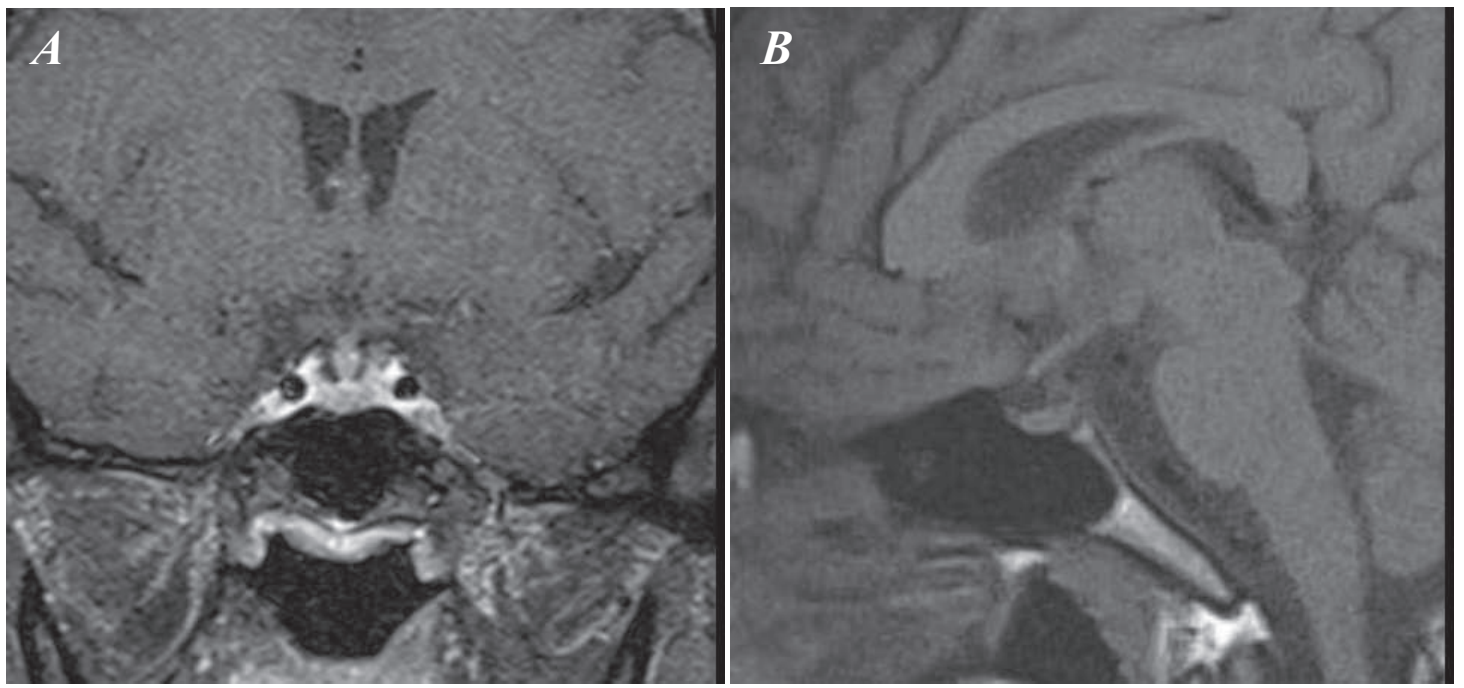

Figure 1. T-1 weighted pituitary MRI of the patient demonstrating $(A)$ Thickening of the pituitary stalk (arrow) $(B)$ The hyperintense signal in the posterior pituitary was not evident (arrow).

ISSN 0857-1074

Copyright $\odot 2011$ by the JAFES

Received January 26, 2011. Accepted March 26, 2011.
Corresponding author: Chng Chiaw Ling, MD

Singapore General Hospital

Outram Road, Singapore 169608

Email:chng.chiaw.ling@singhealth.com.sg 
the other organ systems (posterior pituitary, skin, mucous membranes and bones) made LCH a more likely diagnosis than LAM, which was later confirmed on histology.

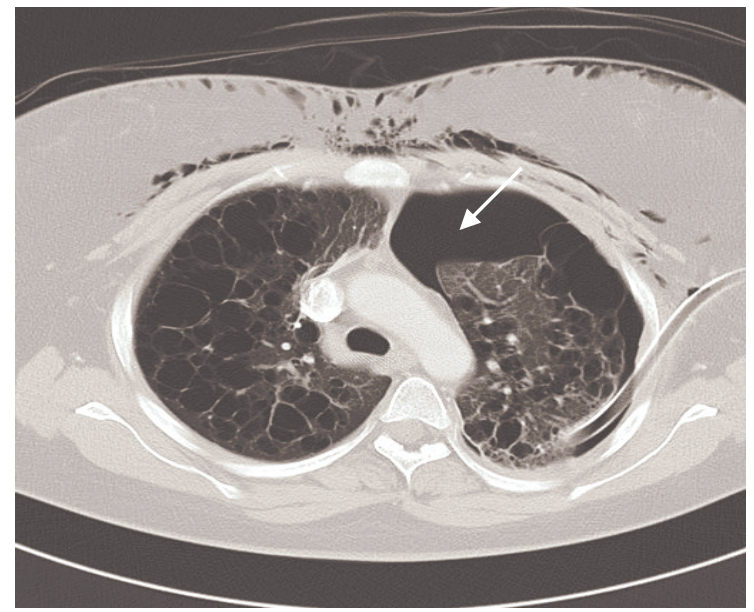

Figure 2. High-resolution CT of the thorax of the patient showing extensive bilateral cystic lesions of the lungs and a left pneumothorax (arrow)
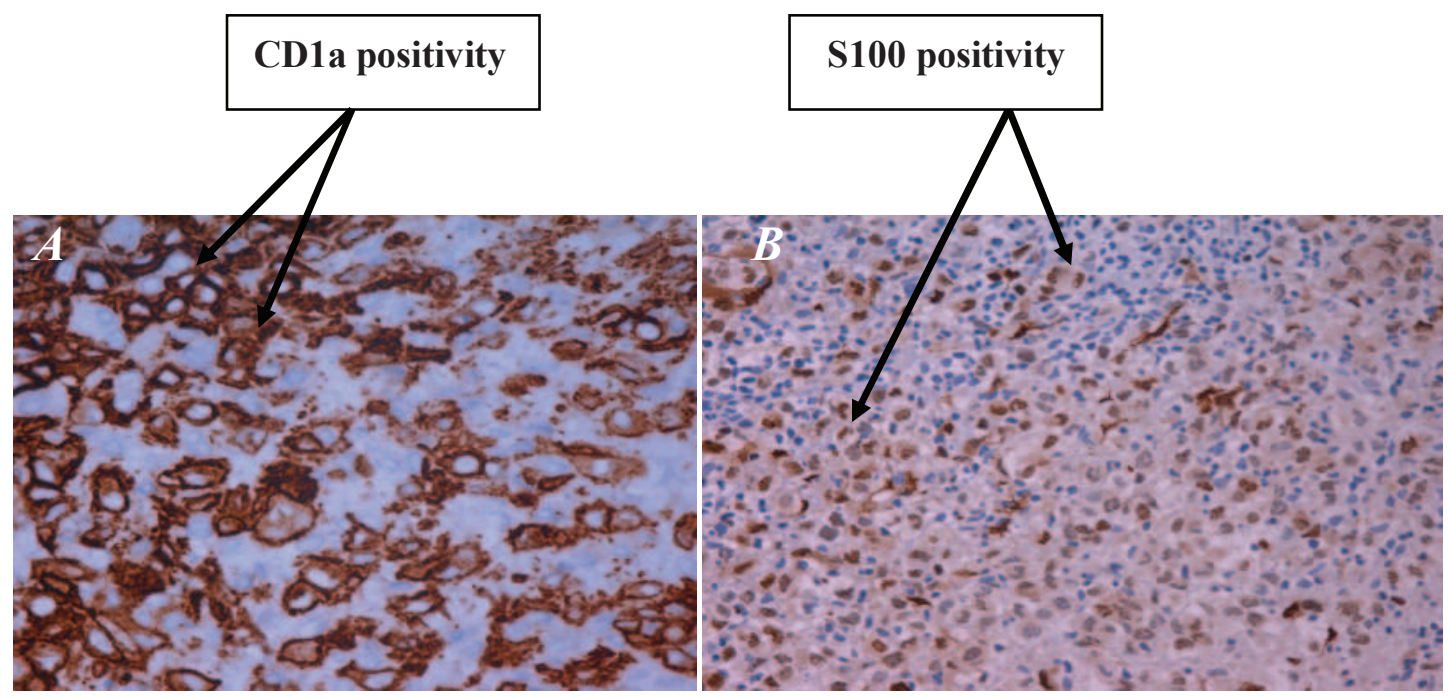

Figure 3. Right axillary biopsy slides (at 40x magnification) demonstrating (A) Diffuse CD1a positive Langerhans cells (B) The Langerhans cells are reactive for $\mathrm{S} 100$

\section{Acknowledgment}

Special thanks to Dr Victor Ng Weng Leong from Department of Pathology, Changi General Hospital for providing the histology slides of this patient.

\section{References}

1. Nezelof C, Frileux-Herbet F, Cronier-Sachot J. Disseminated histiocytosis $\mathrm{X}$ : analysis of prognostic factors based on a retrospective study of 50 cases. Cancer 1979;44:1824-38.

2. Ryu JH, Moss J, Beck GJ et al. The NHLBI lymphangioleiomyomatosis registry: characteristics of 230 patients at enrollment. Am J Respir Crit Care Med 2006;173:105-11.

3. Carsillo T, Astrinidis A, Henske EP. Mutations in the tuberous sclerosis complex gene TSC2 are a cause of sporadic pulmonary lymphangioleiomyomatosis. Proc Natl Acad Sci U S A 2000;97:608590.

4. Astrinidis A, Henske EP. Tuberous sclerosis complex: linking growth and energy signaling pathways with human disease. Oncogene 2005;24:7475-81. 